\title{
Vascular Diseases of the Spinal Cord: A Review
}

Mirjam Rachel Heldner, $M D^{1}$

Marcel Arnold, $M D^{1}$

Krassen Nedeltchev, $M D^{2}$

Jan Gralla, MD, MSC ${ }^{3}$

Jürgen Beck, $M D^{4}$

Urs Fischer, MD, MSc ${ }^{1, *}$

\author{
Address \\ ${ }^{*, 1}$ Department of Neurology, Inselspital, Bern University Hospital and \\ University of Bern, Bern, Switzerland \\ Email: urs.fischer@insel.ch \\ Email: mirjam.heldner@insel.ch \\ Email: marcel.arnold@insel.ch \\ ${ }^{2}$ Department of Neurology, Triemli Hospital, Zurich, Switzerland \\ Email: Krassen.Nedeltchev@triemli.zuerich.ch \\ ${ }^{3}$ Department of Diagnostic and Interventional Neuroradiology, Inselspital, \\ Bern University Hospital and University of Bern, Bern, Switzerland \\ Email: jan.gralla@insel.ch \\ ${ }^{4}$ Department of Neurosurgery, Inselspital, Bern University Hospital and \\ University of Bern, Bern, Switzerland \\ Email: juergen.beck@insel.ch
}

Published online: 9 August 2012

(C) Springer Science+Business Media, LLC 2012

Keywords Vascular diseases of the spinal cord - Acute spinal cord ischemia syndrome (ASCIS) - Acute spinal cord hemorrhage - Vascular malformations of the spinal cord - Current treatment recommendations .

Treatment . Spinal vascular malformations - Thoracic endovascular aortic repair

\section{Opinion statement}

- In acute spinal cord ischemia syndrome (ASCIS), treatment recommendations are derived from data of cerebral ischemic stroke, atherosclerotic vascular disease and acute spinal cord injury. Besides acute management, secondary prevention is of major importance. Pathologies affecting the aorta as well as underlying cerebrovascular conditions should be treated whenever possible.

- ASCIS may occur after aortic surgery, less often after thoracic endovascular aortic repair (TEVAR). Protocols are proposed.

- Acute spinal cord hemorrhage can be treated surgically and/or pharmacologically.

- Symptomatic treatment in patients with a spinal cord lesion is of major importance. Depending on level and extension of the lesion, there is a risk for systemic and neurological complications, which may be life-threatening.

- Each spinal vascular malformation is a unique lesion that needs an individualized treatment algorithm. In case of a symptomatic vascular malformation, endovascular intervention is the primary treatment option. 
- Spinal dural Arteriovenous fistula (AVF) may be treated endovascularly or surgically. If preoperative localization of the fistula is possible, surgery is feasible with a low complication rate. In comparison, endovascular approaches are less invasive.

- Spinal AVM are rather treated endovascularly than surgically or in a stepwise multidisciplinary approach.

- Symptomatic and exophytic spinal cavernous angiomas should be treated surgically. Deep spinal cavernous angiomas that are asymptomatic or only show mild symptoms can be observed.

\section{Introduction}

The 3 major groups of vascular diseases of the spinal cord are acute spinal cord ischemia syndrome (ASCIS), spinal cord hemorrhage, and spinal vascular malformations.

Acute spinal cord ischemia syndrome (ASCIS) (Fig. 1c-f)

Compared with acute cerebrovascular diseases

ASCIS is a rare condition, accounting for $5 \%$ to $8 \%$ of all acute myelopathies and $1 \%$ to $2 \%$ of all vascular neurological pathologies [1, 2]. It is mostly a single event. Main causes of ASCIS are interventions and pathologies affecting the aorta $[3,4 \bullet 5]$. Further etiologies include any pathology that may also cause acute cerebral ischemia, (eg, atherosclerosis, cardiac, and arterio-arterial embolism, infection, hypercoagulable state, and vasculitis). Severe hypotension, cardiac arrest, or rarely, spinal venous pathologies may also lead to ASCIS [1-3]. The clinical spectrum is broad, ranging from complete or incomplete myelopathy, para- or quadriparesis, loss of sensation, especially of pain and temperature, and loss of bladder function. Many patients suffer associated back pain [2]. In contrast to cerebral ischemia, preceding transient ischemic attacks affecting the spinal cord are rare. Symptoms often develop slower than in acute cerebral ischemia, sometimes taking up to several hours [6]. The functional long-term outcome after ASCIS depends mainly on the severity of initial neurological deficits, especially of motor deficits. Poor prognostic factors are female sex, higher age, lack of improvement within the first 24 hours, and affection of the cone. The frequency of recovery to independent gait varies from $11 \%$ to $46 \%, 20 \%$ to $57 \%$ of the patients remain wheelchair bound. Case fatality rates range from
$9 \%$ to $20 \%[1-3,7-9]$. Treatment recommendations concerning acute stage and secondary prevention are derived from cerebral ischemia, atherosclerotic vascular disease, and acute spinal cord injury. Besides acute management, early secondary prevention is important. An underlying pathology should be treated whenever possible [6, $10 \bullet \bullet, 11 \bullet \bullet]$ (Class I).

\section{Spinal cord hemorrhage (Fig. 1i-j)}

Hemorrhage affecting the spinal cord is rare, and is more frequent in men than in women. It may be caused by trauma, anticoagulation, hereditary or acquired bleeding disorders, or bleeding from spinal vascular malformations, spinal artery aneurysms, primary spinal cord tumors (eg, hemangioblastomas or spinal cord metastases). Rarely it is spontaneous and in many cases the etiology remains undiscovered. The hemorrhage can be intramedullary, subarachnoidal, subdural, or epidural [12]. Hematomyelia is caused by bleeding within the spinal cord. The blood tends to dissect longitudinally above and below the hemorrhage, disrupting gray matter more than white matter [13]. Spinal subarachnoidal hemorrhage accounts for less than $1 \%$ of all subarachnoidal hemorrhages. Spinal epidural hemorrhage occurs at least 4 times more commonly than spinal subdural hemorrhage. Patients with spinal cord hemorrhage usually present with acute symptoms with back or neck pain that may be intense, knife-like, and often show a radicular component. Patients with spinal subarachnoidal hemorrhage may additionally show symptoms resembling meningitis, such as meningeal irritation with headache, neck stiffness, disturbance of consciousness and epileptic seizures and are also often misdiagnosed as having cerebral 

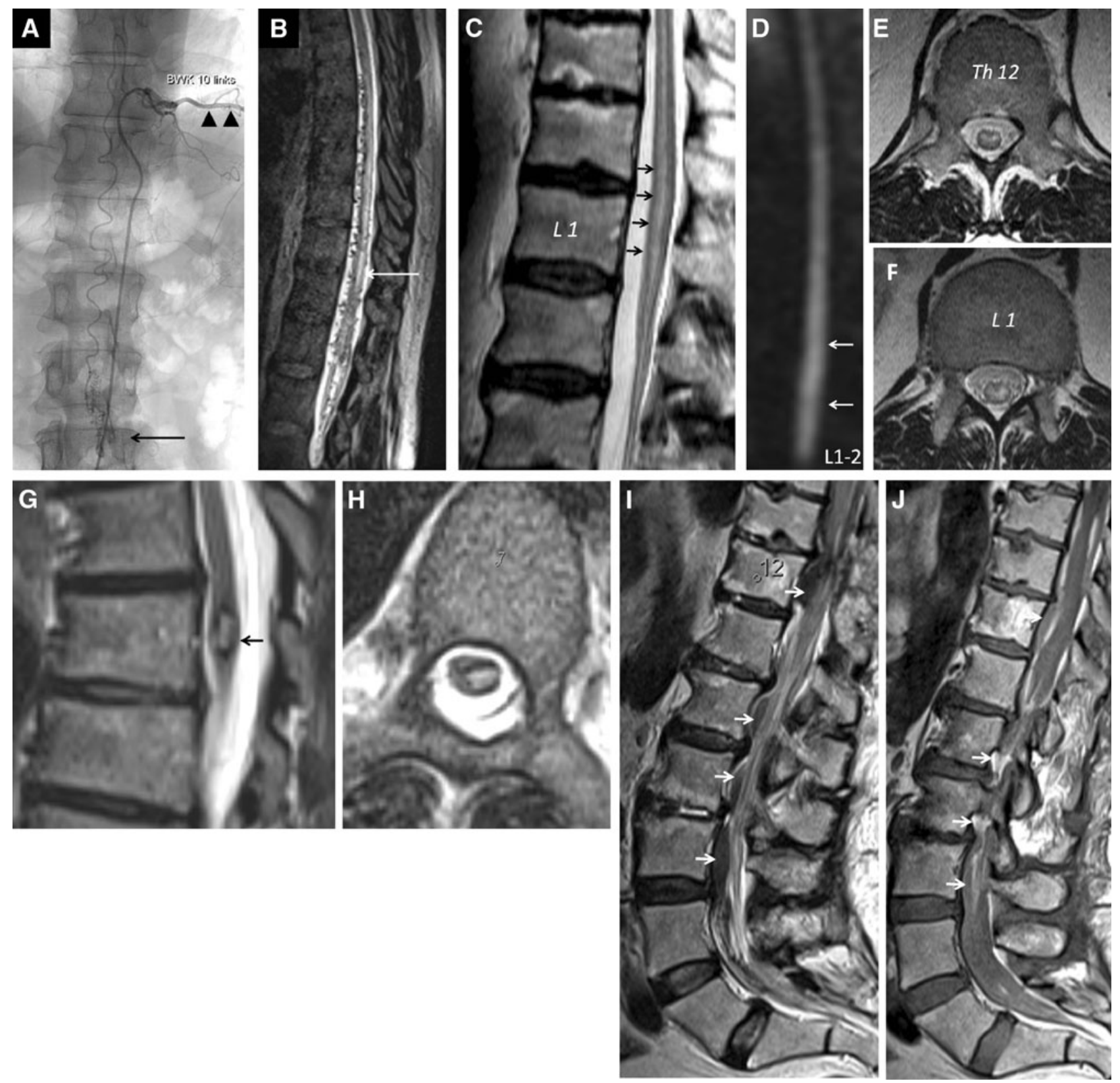

Figure 1. a b A 50-year-old man with recurrent extensive spinal intradural arteriovenous malformation. a Catheter angiography showing the artery of Adamkiewicz originating from the left intercostal artery at the Th10 level (black arrowheads). The anterior spinal artery is rather tortuous. The vessel convolute (nidus) is seen at the level L2-3 (black arrow). b High resolution sagittal heavy T2-weighted (CISS) MRI reveals extensive worm-like intraspinal vessels in the thoracolumbar region. Notice the myelopathy signal (white arrow). c-f A 72-year-old man with cramp-like pains in the thighs and the calves. MRI showing evidence of spinal cord ischemia. $\mathbf{c}$ Notice the T2 bright signal within the spinal cord from the Th11 level down to the conus medullaris. d Sagittal diffusion-weighted image showing abnormal signal in the affected part of the spinal cord. Axial T2weighted images show the possible signal abnormality pattern. e Snake-eyes (or snake-bite) pattern at Th12. $\mathbf{f H}$-like pattern at L1. $\mathbf{g}$-h Intramedullary cavernous angioma at the Th7 level. Sagittal $\mathbf{g}$ and axial $\mathbf{h}$ T2-weighted images. Notice the bright internal signal surrounded by the dark hemosiderin ring. $\mathbf{i}-\mathbf{j}$ Intraspinal perimedullary extensive hemorrhage, often difficult to detect. Sagittal T2- $\mathbf{i}$ and T1-weighted $\mathbf{j}$ images showing low and bright signal of the hemorrhage (arrows). 
hemorrhage [12]. Specific treatment of acute spinal cord hemorrhage is surgical and/or pharmacological [12, 13].

\section{Spinal vascular malformations}

Most frequent spinal vascular malformations, causing hemodynamic changes of the spinal cord, are classified into different types, depending on their location and vascular pathology: spinal dural arteriovenous fistulas (AVF), arteriovenous malformations (AVM), and cavernous angiomas. Dural arteriovenous fistula (AVF) is the most common type of spinal vascular malformations. Spinal dural AVF are more common in men, usually presenting after the forth decade and are probably acquired. The etiology of spinal dural AVF is unknown. The clinical presentation is mostly a slowly progressive, often step-wise and rarely acute myeloradiculopathy. A venous congestion caused by arterial overload of venous drainage due to the fistula, initiates edema and mylopathy of the spinal cord leading to a broad range of clinical symptoms. Spinal cord hemorrhage is almost never seen in patients with spinal dural AVF. Occlusion of the fistula by surgery or endovascular embolization is indicated and can improve or at least stabilize the clinical condition [2, 14-18]. Early recognition of spinal dural AVF with subsequent aggressive treatment may best prevent further neurologic decline.
Having this mostly vascular entity in mind and initiating spinal imaging early, may significantly impact patient's outcome [19].

The second most common vascular malformation of the spinal cord is the spinal arteriovenous malformation (AVM) (Fig. 1a-b). Clinical symptoms are usually first seen in adolescence or early adulthood. Damage of the spinal cord is most often caused by acute intramedullary or subarachnoidal hemorrhage, venous congestion, and less frequently by mass or steal effect. Spinal AVM are rather treated endovascularly than surgically or in a stepwise multidisciplinary approach $[2,15,18,20]$.

The third most common vascular malformation of the spinal cord is the spinal cavernous angioma (Fig. 1g-h). It can cause a transverse spinal cord syndrome with acute worsening by hemorrhage or mass effect. Most patients present with progressive or stepwise clinical deterioration which is thought to be due to gliosis, microthrombosis, microcirculatory changes, and repeated minor bleedings into the spinal cavernous angioma. Acute and severe neurological symptoms can occur due to hemorrhage into the spinal cord [21]. Spinal cavernous angiomas may be treated surgically when indicated $[2,22,23]$. Most symptomatic or neurologically declining patients with spinal cavernous angioma are surgical candidates $[21,24]$.

\section{Treatment}

There are no prospective or randomized studies comparing different treatment options in patients with ASCIS, acute spinal cord hemorrhage, or spinal vascular malformations. Therefore, scientific evidence on treatment of vascular diseases of the spinal cord is limited. A large multicenter study would be needed to gain enough statistical power in these rare diseases.

\section{Treatment of acute spinal cord ischemia syndrome (ASCIS)}

Treatment recommendations of ASCIS concerning acute stage and secondary prevention are derived from acute cerebral ischemia, atherosclerotic vascular disease, and acute spinal cord injury. Besides acute management, secondary prevention is of major importance. An underlying pathology (eg, systemic vasculitis, aortic pathology, cardiogenic embolism, hypovolemia, anemia) should be treated whenever possible $[6,10 \bullet \bullet$, $11 \bullet \bullet$ ] Class I). 


\section{Pharmacological treatment}

\section{Acetylsalicylic acid}

\begin{tabular}{|c|c|}
\hline & $\begin{array}{l}\text { As for acute treatment of cerebral ischemia acetylsalicylic acid is used for } \\
\text { ASCIS in most centers }[10 \bullet \bullet] \text { (Class I). }\end{array}$ \\
\hline Standard dosage & $\begin{array}{l}\text { We administer } 250 \mathrm{mg} \text { up to } 500 \mathrm{mg} \text { intravenously in the acute stage, fol- } \\
\text { lowed by } 100 \mathrm{mg} \text { oral per day for secondary prevention. The AHA guidelines } \\
\text { recommend the following dosages: In the acute stage: } 325 \mathrm{mg} \text { oral, for sec- } \\
\text { ondary prevention: } 50 \mathrm{mg} \text { up to } 325 \mathrm{mg} \text { oral }[10 \bullet \bullet, 11 \bullet \bullet] \text { (Class I). }\end{array}$ \\
\hline Contraindications & $\begin{array}{l}\text { Hypersensitivity to salicylates and/or other anti-inflammatory drugs, } \\
\text { history of gastrointestinal hemorrhage, or bleeding diathesis, gastroin- } \\
\text { testinal ulcera, severe liver insufficiency, severe renal insufficiency, last } \\
\text { trimenon of pregnancy. }\end{array}$ \\
\hline Main drug interaction & $\begin{array}{l}\text { Increased bleeding risk when administered with other platelet inhibitory or } \\
\text { anticoagulant drugs. }\end{array}$ \\
\hline Main side effects & $\begin{array}{l}\text { Abdominal pain, gastrointestinal hemorrhage, or other bleeding, hypersen- } \\
\text { sitivity reactions. }\end{array}$ \\
\hline \multicolumn{2}{|c|}{ Acetylsalicylic acid and dipyridamol or clopidogrel } \\
\hline & $\begin{array}{l}\text { Instead of acetylsalicylic acid, a combination therapy of acetylsalicylic } \\
\text { acid and dipyridamol may be administered. In case of occurrence of } \\
\text { ASCIS in patients already on treatment with acetylsalicylic acid, or in case } \\
\text { of hypersensitivity to acetylsalicylic acid, oral clopidogrel may be used. In } \\
\text { the acute stage we usually administer } 75 \mathrm{mg} \text {, or a loading dose of } \\
300 \mathrm{mg} \text {, followed by } 75 \mathrm{mg} \text { (according to AHA guidelines) per day for } \\
\text { secondary prevention. } \\
\text { In the long term, daily administration of acetylsalicylic acid alone or } \\
\text { together with dipyridamol, or clopidogrel alone reduce the risk of secondary } \\
\text { vascular events and death }[10 \bullet \bullet, 11 \bullet \bullet] \text { (Class I). }\end{array}$ \\
\hline \multicolumn{2}{|c|}{$\begin{array}{l}\text { Thrombolytic therapy (eg, alteplase; tissue plasminogen } \\
\text { activator) }\end{array}$} \\
\hline
\end{tabular}

Thrombolytic therapy has been successfully used in few published case reports of ASCIS [5, 25]. However, randomized controlled trials on thrombolysis in patients with ASCIS are lacking. Therefore, thrombolysis remains experimental. Before initiation of thrombolysis, the diagnosis has to be certain and contraindications have to be excluded. This may lead to a delay beyond the established treatment window for intravenous thrombolysis of 4.5 hours, derived from evidence in patients with acute cerebral ischemia.

Standard dosage of alteplase as in cerebral ischemia

Contraindications
$0.9 \mathrm{mg} / \mathrm{kg}$ (maximum dose $90 \mathrm{mg}$ ), first $10 \%$ administered as bolus within 1 minute and the remaining over 1 hour $[26,27]$ (Class I).

First symptoms more than 4.5 hours ago, hypersensitivity to the substance, acute intra- or extracranial bleeding, and/or bleeding less than 3 months ago, vascular malformations, aortic dissection, traumatic brain injury less than 3 months ago, surgery, or biopsy of a parenchymatous organ, gastrointestinal bleeding, pregnancy and birth less than 30 days ago, arterial tap in a noncompressible location less than 7 days ago, infectious endocarditis, 
Main drug interactions

assumed septic embolus, hemorrhagic diathesis or coagulopathy, arterial hypertension, that cannot be lowered (>185 mm Hg systolic and/or $>100 \mathrm{~mm} \mathrm{Hg}$ diastolic), severe accompanying disease, INR $>1.7$, thrombocytes $<100 \mathrm{G} / \mathrm{l}$, etc. [26, 27] (Class I).

Increased bleeding risk when administered with anticoagulant or platelet inhibitory drugs. Orolingual angioedema and anaphylaxis may occur, usually when receiving concomitant angiotensin-converting enzyme (ACE) inhibitors.

Main side effects Bleeding.

Systemic corticosteroids

Treatment with systemic corticosteroids has not been studied in patients with ASCIS apart from case reports. Furthermore, administration of

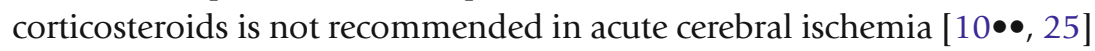
(Class I). Therefore, treatment with corticosteroids is not recommended in patients with ASCIS.

\section{Specific treatment with thoracic endovascular aortic repair (TEVAR)}

ASCIS that occurs after aortic surgery is a feared complication. In repair involving thoracic endovascular aortic repair (TEVAR) the risk of ASCIS appears to be lower than for open procedures. Moreover, protocols for the prevention and treatment of delayed postinterventional ASCIS have been developed $[4 \bullet, 28,29]$ (Class III). These protocols seem to be effective in reversing and limiting the neurological deficits and consist of an immediate combination of blood pressure support by volume and/or vasopressor agents and an immediate reduction of spinal cord canal pressure with lumbar drains. These protocols have not yet been evaluated in a controlled study in ASCIS and in ASCIS due to other causes than aortic interventions. The benefit in other causes is questionable especially because of the time delay from symptom onset to clinical presentation.

\section{Treatment of acute spinal cord hemorrhage}

\section{Pharmacological treatment}

There are only limited pharmacological options to treat spinal cord hemorrhage. Vitamin K, fresh frozen plasma, and prothrombin complex may be used for hemorrhages induced by vitamin $\mathrm{K}$ antagonists, protamin sulfate for heparin-induced hemorrhages, platelet transfusions for thrombocytopenia, specific clotting factor concentrates or, fresh frozen plasma for clotting factor deficiencies as hemophilia A and hemophilia B (Christmas disease) [30]. Other specific pharmacological treatment is not proven.

\section{Nonpharmacological treatment}

The aim of immediate surgery in patients with acute spinal cord hemorrhage is stopping of the bleeding, decompression of neural structures and removal of the hematoma. Surgery may be indicated for hematomyelia [13]. In 
patients with subdural hemorrhage, surgery is often necessary as it is mostly the case in patients with epidural hemorrhage $[12,31]$. The milder the preoperative symptoms and the quicker surgical decompression can be performed, the better are the chances for complete recovery [12]. A report of few patients with spontaneous spinal epidural hemorrhages showed an improvement of symptoms even if surgery was performed not earlier than 36 hours after symptom onset [32]. However, there are some reports in patients with epidural hemorrhage that favor a conservative approach in the following situations: Either in case of improving symptoms between symptom onset and diagnosis or in case of a longitudinally extending, less restricted compressive hematoma [31, 33]. Treatment of spinal subarachnoid hemorrhage and hematoma depends on etiology and might be an urgent intervention for decompression. Underlying spinal arteriovenous malformations should be treated as listed below. Conservative treatment with bed rest is a treatment option for patients with spinal subarachnoid hemorrhage and hematoma with stable and minimal neurological impairment [34].

\section{Acute symptomatic treatment of ASCIS and acute spinal cord hemorrhage}

Within the first days up to weeks of hospitalization, patients with a spinal cord lesion are at risk for several systemic as well as neurological complications. These complications may be life-threatening. Early intervention is necessary. Intensive care unit admittance for strict monitoring of vital signs and neurological status is necessary in patients with a cervical or a high thoracic lesion $[35 \bullet]$.

\section{Cardiovascular complications}

Cardiovascular complications are often caused by neurogenic shock, which leads to arterial hypotension, usually accompanied by bradycardia. Hemodynamic instability may be related to the underlying etiology. Strict blood pressure control is necessary to maintain adequate perfusion. A combination of volume resuscitation and the use of vasopressors with chronotropic and inotropic properties (norepinephrine, dopamine) are treatment options. Continuous blood pressure monitoring is recommended. Bradycardia is frequently seen in high cervical (C1 up to C5) lesions. External pacing or administration of atropine may be needed. Autonomic dysreflexia, eg, episodic paroxysmal hypertension to very high levels with headache, tachycardia or bradycardia, flushing, sweating and anxiety/agitation may be seen in cervical, and upper thoracic cord lesions, though autonomic dysreflexia was originally described after traumatic spinal cord injury. The complication of autonomic dysreflexia is dramatic and life-threatening. It is thought to arise from inappropriate release of catecholamines from adrenal glands in response to visceral stimuli. Therefore, treatment aims at relieving stimuli on bowel and bladder decompression in order to control blood pressure. Arterial vasodilators and intravenous benzodiazepines can be given to control blood pressure [36]. 


\section{Respiratory complications}

Chest physiotherapy should be installed as soon as possible. Attention to the respiratory system should especially be given in patients with higher cervical but also thoracic lesions. Respiratory complications as pneumonia, pulmonary embolism, pulmonary edema, and respiratory failure may occur. Increased respiratory rate, declining forced vital capacity, rising $\mathrm{pCO}_{2}$, or falling $\mathrm{pO}_{2}$ are signs of impending respiratory failure, and indicate urgent intubation and pressure support ventilation.

\section{Venous thrombo- and pulmonary embolism}

The risk of thromboembolic disease, including deep venous thrombosis (DVT) and pulmonary embolism in patients with vascular diseases of the spinal cord is increased, mainly due to immobilization and to decreased muscle contraction because of paresis. Low-molecular-weight heparin (LMWH) is considered the treatment of choice besides mechanical means and mobilization. LMWH is recommended as soon as primary hemostasis is achieved and the risk of bleeding or re-bleeding is low $[35 \bullet, 37 \bullet \bullet, 38 \bullet]$.

\section{Urinary catheterization}

Prevention and treatment of urinary tract infections is important. On admission, urinary catheterization should be installed to avoid bladder distension. In the long term, intermittent straight catheterization to decompress the urinary bladder is preferable, due to a higher risk of bladder infections with an in-dwelling urinary catheter.

\section{Pain control}

Adequate pain management is important. In addition to nonsteroidal inflammatory and opioid agents, the use of pregabalin, gabapentin, or duloxetin to treat neuropathic pain may be indicated. Antispasmodics may also be helpful $[35 \bullet]$.

\section{Temperature control}

An optimal ambient temperature should be maintained, as patients with spinal cervical cord lesion might have difficulties controlling their body temperature, secondary to an inability to sweat below the level of the lesion.

\section{Gastrointestinal stress ulceration}

Especially patients with cervical cord lesions are at high risk for stress ulceration and should receive prophylaxis with proton pump inhibitors.

\section{Pressure sores}

Pressure sores, especially on the buttocks and heels, can develop quickly in immobilized patients. For prevention, patients have to be 
turned side to side (log-rolled) regularly, every 2 to 4 hours. Special mattresses can also be used.

\section{Functional recovery}

Early mobilization is important for good lung and cardiovascular function, and the prevention of pressure ulcers and venous stasis. Physiotherapy and occupational therapy should be started as soon as possible. If needed, psychological support and social workers might be beneficial.

\section{Long-Term symptomatic treatment of ASCIS and acute spinal cord hemorrhage}

Often, patients after ASCIS and after acute spinal cord hemorrhage suffer from residual deficits which are causing secondary complications including bladder, bowel, and sexual dysfunction, spasticity, and chronic pain. These conditions have to be managed [35•].

\section{Treatment of spinal vascular malformations}

Each spinal vascular malformation is a unique lesion that needs an individualized treatment algorithm. Due to few data, the natural history of an asymptomatic incidental finding is difficult. It is generally accepted that symptomatic vascular malformations should be treated whenever possible. In case of an assumed high treatment risk, an observation of the spinal dural AVM, AVF, or the spinal dural cavernous angiomas is warrantable in individual cases. Spinal dural AVF should be treated without delay, since short- and medium-term prognosis are poor and chances of favourable outcome after treatment are good [16, 18] (Class III).

Treatment should only be performed in specialized centers with vascular neurosurgeons, interventional neuroradiologists, and neurologists.

\section{Pharmacological treatment}

There are no drugs that have the capacity to affect or heal vascular malformations. Steroids may be helpful for a short period to improve the patient's symptoms by reducing vasogenic edema. However, steroids should only be prescribed for a short period because of their adverse systemic short- and longterm effects (eg, immunosuppression, hyperglycemia, gastric ulceration, and psychosis) [30].

A successful occlusion of a spinal dural AVF leads to immediate hemodynamic changes of the dilated intrathecal veins. Therefore, an additional heparinization for prevention of peri- and postinterventional thrombosis of the dilated veins is recommended [39].

\section{Interventional procedures}

The possible interventional approach is endovascular embolization [40••]. Spinal AVM can be treated by embolization with liquid embolization material, rarely particles or coils $[15,18]$ (Class IV). Spinal AVM are rather treated endovascularly than surgically. The benefit of endovascular treatment of 
spinal dural AVF compared with surgical treatment is that it is less invasive, preserving tissue and, therefore, function of the surrounding spinal cord. However, there is a relevant percentage of spinal dural AVF that are technically not achievable endovascularly, even not in specialized centers. If there is no complete occlusion of the venous side, then collaterals of the neighbouring segmental arteries are to expect with consecutively missing improvement or progression of symptoms and deficits. Therefore, after endovascular treatment, regular clinical and imaging follow-ups are needed $[14-16,18,39,41]$.

\section{Surgery}

Possible surgical treatment options are ligation, disconnection, or resection. Spinal AVM, AVF, and spinal cavernous angiomas may be treated surgically. Preoperative localization of the fistula is often recommended, however, surgical treatment spinal dural of AVF may be the first treatment choice. After opening of the dura, the draining radiculomedullary vein has to be occluded. This leads to a complete disconnection of the spinal dural AVF $[17,41]$. In case of multiple sites of fistula formation, surgery has the advantage of direct visibility of all feeding vessels. Surgical disconnection of spinal dural AVF is a rather safe and straightforward surgical procedure at specialized centers with favorable long-term improvement of symptoms. Furthermore, surgery is the only option, if the arterial feeding vessel is impossible to access endovascularly because of tortuous vascular anatomy, or if the vessel supplies blood to healthy regions of the spinal cord.

Surgery is the treatment option of spinal cavernous angiomas [22]. The indication for surgery must carefully weight surgical risks against the natural history of the disease. The risk for permanent neurological decline after surgery is estimated to be $6 \%$ to $12 \%$ with most patients ( $44 \%$ to $80 \%$ ) showing improvement after surgical resection [24]. Since current available data indicate bleeding rates of $2.5 \%$ to $4.5 \%$ per year, most centers treat - (eg, surgically resect spinal cavernous angiomas) $[21,24]$. There are few data that report about the lack of rebleeding with a conservative approach [23]. Symptomatic and exophytic spinal cavernous angiomas should be excised whereas deep intrinsic spinal cavernous angiomas with only mild symptoms should be observed [21].

\section{Combined interventional and surgical therapy}

Current optimum treatment can only be reached by case discussion and an individualized treatment plan that is established by an interdisciplinary team of specialized vascular neurosurgeons, interventional neuroradiologists, and neurologists on a case by case basis in neurovascular centers.

\section{Radiation therapy}

Radiation therapy is still sometimes applied to spinal AVM and spinal cavernous angiomas. In our opinion it may rarely be a treatment option in few spinal AVM, but is, especially in spinal cavernous angiomas, no longer an acceptable treatment option because of the high radiation sensibility of the myelon. 


\section{Acknowledgment}

The authors thank Marwan El-Koussy for providing the figures.

\section{Disclosures}

No potential conflicts of interest relevant to this article were reported.

\section{References and Recommended Reading}

Papers of particular interest, published recently, have been highlighted as:

- Of importance

- Of major importance

1. Nedeltchev K, Loher TJ, Stepper F, et al. Long-term outcome of acute spinal cord ischemia syndrome. Stroke. 2004;35:560-5.

2. Mumenthaler M, Mattle $H$, editors. Neurologie 12th ed. Stuttgart: Georg Thieme Verlag, 2008; pp 363-7.

3. Cheshire WP, Santos CC, Massey EW, et al. Spinal cord infarction: etiology and outcome. Neurology. 1996;47:321.

4. $\quad$ Messé SR, Bavaria JE, Mullen M, et al. Neurologic outcomes from high risk descending thoracic and thoracoabdominal aortic operations in the era of endovascular repair. Neurocrit Care. 2008;9:344.

This paper shows the benefit of TEVAR in descending thoracic and thoracoabdominal aortic interventions.

5. Restrepo L, Guttin JF. Acute spinal cord ischemia during angiography treated with intravenous thrombolytic therapy. Tex Heart Inst J. 2006;33:74.

6. Transverse Myelitis Consortium Working Group. proposed diagnostic criteria and nosology of acute transverse myelitis. Neurology. 2002;59:499-505.

7. Geldmacher DS, Bowen BC. Spinal cord vascular disease. In: Bradley WG, Daroff RB, Fenichel GM, Marsden $\mathrm{CD}$, editors. Neurology in clinical practice principles of diagnosis and management. 4th ed. Philadelphia: Butterworth-Heimann; 2004. p. 1313-22.

8. Masson C, Pruvo JP, Meder JF, et al. Spinal cord infarction: clinical and magnetic resonance imaging findings and short term outcome. J Neurol Neurosurg Psychiatry. 2004;75:1431.

9. De Seze M, De Seze M, Joseph PA, et al. Functional prognosis of paraplegia due to cord ischemia: a retrospective study of 23 patients. Rev Neurol (Paris). 2003;159:1038-45.

10.• Adams Jr HP, del Zoppo G, Alberts MJ, et al. Guidelines for the early management of adults with ischemic stroke: a guideline from the American Heart Association/American Stroke Association Stroke Council, Clinical Cardiology Council, Cardiovascular Radiology and Intervention Council, and the Atherosclerotic Peripheral Vascular Disease, and Quality of Care Outcomes in Research Interdisciplinary Working Groups: The American Academy of Neurology affirms the value of this guideline as an educational tool for neurologists. Circulation. 2007;115:e478.

This paper gives a good overview concerning management of acute ischemic stroke.

11.• Furie KL, Kasner SE, Adams RJ, et al. Guidelines for the prevention of stroke in patients with stroke or transient ischemic attack: a guideline for healthcare professionals from the American Heart Association/ American Stroke Association. Stroke. 2011;42:227-76.

This paper gives a good overview concerning prevention of stroke in patients with stroke or transient ischemic attack.

12. Kreppel D, Antoniadis G, Seeling W. Spinal hematoma: a literature survey with meta-analysis of 613 patients. Neurosurg Rev. 2003;26:1-49.

13. Leep Hunderfund AN, Wijdicks EF. Intramedullary spinal cord hemorrhage (hematomyelia). Rev Neurol Dis. 2009;6:E54-61.

14. Lev N, Maimon S, Rappaport ZH, et al. Spinal dural arteriovenous fistulae: a diagnostic challenge. Isr Med Assoc J. 2001;3:492.

15. Krings T, Lasjaunias $P$, Reinges MHT, et al. Spinal vascular malformations. Diagnostic and therapeutic management. Clin Neuroradiol. 2006;4:217-27.

16. Van Dijk JM, TerBrugge KG, Willinsky RA, et al. Multidisciplinary management of spinal dural arteriovenous fistulas. Clinical presentation and longterm follow-up in 49 patients. Stroke. 2002;33:1578-83.

17. Huffmann BC, Gilsbach JM, Thron A. Spinal dural arteriovenous fistulas: a plea for neurosurgical treatment. Acta Neurochir. 1995;135:44-51. 
18. Berenstein A, Lasjaunias P, TerBrugge KG. Clinical and endovascular treatment aspects in adults. In: Berenstein A, Lasjaunias P, TerBrugge KG, editors. Surgical Neuroangiography 2.2. Berlin: Springer; 2004. p. 737-872.

19. Fugate JE, Lanzino G, Rabinstein AA. Clinical presentation and prognostic factors of spinal dural arteriovenous fistulas: an overview. Neurosurg Focus. 2012;32:E17.

20. Bemporad JA, Sze GS. MR imaging of spinal cord vascular malformations with an emphasis on the cervical spine. Magn Reson Imaging Clin N Am. 2000;8:581.

21. Gross BA, Du R, Popp AJ, Day AL. Intramedullary spinal cord cavernous malformations. Neurosurg Focus. 2010;29:E14.

22. Zevgaridis D, Medele RJ, Hamburger C, et al. Cavernous haemangiomas of the spinal cord. A review of 117 cases. Acta Neurochirurgica. 1999;141:237-45.

23. Kharkar S, Shuck J, Conway J, Rigamonti D. The natural history of conservatively managed symptomatic intramedullary spinal cord cavernomas. Neurosurgery. 2007;60:865-72.

24. Wachter D, Psychogios M, Gilsbach JM, Rohde V. Spinal cord cavernoma-operative strategy and results in 30 patients. J Neurol Surg A Cent Eur Neurosurg. 2012;73:125-31.

25. Baba H, Tomita K, Kawagishi T, Imura S. Anterior spinal artery syndrome. Int Orthop. 1993;17:353.

26. The National Institute of Neurological Disorders and Stroke rt-PA Stroke Study Group: tissue plasminogen activator for acute ischemic stroke. N Engl J Med. 1995;333:1581-1587.

27. Hacke W, Kaste M, Bluhmki E, et al. Thrombolysis with alteplase 3 to 4.5 hours after acute ischemic stroke. N Engl J Med. 2008;359:1317-29.

28. Grabenwöger M, Alfonso F, Bachet J, et al. Thoracic Endovascular Aortic Repair (TEVAR) for the treatment of aortic diseases: a position statement from the European Association for Cardio-Thoracic Surgery (EACTS) and the European Society of Cardiology (ESC), in collaboration with the European Association of Percutaneous Cardiovascular Interventions (EAPCI). Eur J Cardiothorac Surg. 2012; (Epub ahead of print).

29. McGarvey ML, Cheung AT, Szeto W, Messé SR. Management of neurologic complications of thoracic aortic surgery. J Clin Neurophysiol. 2007;24:336.

30. Lüllmann $\mathrm{H}$, Mohr $\mathrm{K}$, Wehling $\mathrm{M}$, Lüllmann $\mathrm{H}$, Mohr K, Wehling M. editors. Stuttgart: Thieme, $15^{\text {th }}$ edition. Pharmacol Toxicol. 2003;17784:370-6.
31. Groen RJM. Non-operative treatment of spontaneous spinal epidural hematomas: a review of the literature and a comparison with operative cases. Acta Neurochir (Wien). 2004;146:103-10.

32. Daentzer D, Boker DK. Spontaneous spinal hemorrhage. Outcome after surgical therapy of epidural hematomas. Der Nervenarzt. 2000;71:116-22.

33. Duffill J, Sparrow OC, Millar J, Barker CS. Can spontaneous spinal epidural haematoma be managed safely without operation? A report of 4 cases. J Neurol Neurosurg Psychiatry. 2000;69:816-9.

34. Domenicucci M, Ramieri A, Paolini S, et al. Spinal subarachnoid hematomas: our experience and literature review. Acta Neurochir. 2005;147:74150 .

35. Markandaya M, Stein M, Menaker J. Acute treatment options for spinal cord injury. Curr Treat Options Neurol. 2012;14:175-87.

Even though this paper primarily addresses traumatic spinal cord injury, many management recommendations may be applied to ASCIS and acute spinal cord hemorrhage as well.

36. Bycroft J, Shergill IS, Chung EA, et al. Autonomic dysreflexia: a medical emergency. Postgrad Med J. 2005;81:232.

37.• Geerts WH, Bergquist D, Pineo GF, et al. Prevention of venous thromboembolism: American College of Chest Physicians Evidence-Based Clinical Practice Guidelines (8th edition). Chest. 2008;133:381S-453S.

This paper provides evidence-based guidelines for the use of thromboprophylaxis in a variety of clinical situations.

38. Raslan AM, Fields JD, Bhardwaj A. Prophylaxis for venous thrombo-embolism in neurocritical care: a critical appraisal. Neurocrit Care. 2010;12:297309.

This paper provides a critical appraisal concerning the use of thromboprophylaxis including in ASCIS.

39. Niimi Y, Berenstein A, Setton A, Neophytides A. Embolization of spinal dural arteriovenous fistulae: results and follow-up. Neurosurgery. 1997;40:67582. discussion 682-3.

40.• Krings T, Thron AK, Geibprasert S, et al. Endovascular management of spinal vascular malformations. Neurosurg Rev. 2010;33:1-9.

This paper discusses interventional and surgical management of spinal vascular malformations.

41. Behrens S, Thron A. Long-term follow-up and outcome in patients treated for spinal dural arteriovenous fistulae. J Neurol. 1999;246:181-5. 